\title{
Imaging of Hip Arthroplasty
}

\author{
Jan Fritz, MD ${ }^{1}$ Brett Lurie, MBBS ${ }^{1}$ Theodore T. Miller, MD, FACR ${ }^{1}$
}

1 Department of Radiology and Imaging, Hospital for Special Surgery, Weill Cornell Medical College, New York, New York

Address for correspondence Theodore T. Miller, MD, FACR,

Department of Radiology and Imaging, Hospital for Special Surgery,

Semin Musculoskelet Radiol 2013;17:316-327.

535 E 70th St, New York, NY 10021 (e-mail: millertt@hss.edu).
Abstract
Keywords
- hip
- arthroplasty
- ALVAL
- adverse tissue reaction
- MR imaging

Imaging studies are central to the evaluation of persistent or recurrent symptoms after hip arthroplasty. The evaluation starts with radiographs and may be followed by arthrography, aspiration, scintigraphy, sonography, computed tomography, and MR imaging. Common etiologies of a painful or dysfunctional hip arthroplasty are mechanical loosening, polyethylene wear-induced osteolysis, adverse local tissue reaction to metal wear products, infection, fractures, heterotopic ossification, tendinopathy, and nerve injury. MR imaging with optimized protocols and dedicated techniques for metal artifact reduction is the most comprehensive imaging modality. In this article, we discuss and illustrate the imaging appearances of these conditions with a focus on the MR imaging evaluation.
Hip arthroplasty is one of the most successful surgical procedures, achieving pain relief, restoring hip joint function, and improving the quality of life in up to $90 \%$ of patients with end-stage degenerative hip disease. ${ }^{1}$ Although developments in bearing materials, implant design, and fixation techniques have continuously increased implant survivorship and patient satisfaction, pain after hip arthroplasty occurs in 0.5 to $40 \%$ of patients. ${ }^{2}$ Imaging studies are central to the evaluation of pain following arthroplasty. In this article, we discuss the imaging appearances of a variety of conditions that may occur following arthroplasty with a focus on the MR imaging evaluation.

\section{Imaging Modalities}

Radiography is the primary modality for the initial evaluation of the painful hip arthroplasty as well as for routine surveillance. Immediately following surgery, anteroposterior radiographs are routinely obtained to determine the position of the arthroplasty and to detect early surgical complications such as periprosthetic fracture. Baseline radiographs are subsequently obtained in at least two projections for future comparison.

Ultrasound is not hampered by artifacts related to nearby metal and is therefore well suited for the assessment of joint effusions, synovial thickening, tissue hyperemia, periarticular collections, tendons, and bursae. ${ }^{3}$ Ultrasound can be used to guide joint aspiration and is the modality of choice to guide percutaneous synovial biopsy.

Fluoroscopically guided joint puncture can be used to obtain fluid for microbiological and cytological analysis, or to guide injection of contrast to assess for communication of the joint with a soft tissue collection or a sinus tract to the skin surface. Contrast material insinuating along a bone-implant or bone-cement interface indicates bone resorption.

Computed tomography (CT) is an accurate modality for the assessment of osteolysis, implant position, hardware integrity, wear, fractures, heterotopic ossification, hematomas, and fluid collections. Metal artifacts can be reduced by increasing the peak voltage and tube charge, using the smallest possible collimation, extending the display CT scale, and repositioning the patient so that the X-ray beam traverses the smallest possible cross-sectional area of the implant. ${ }^{4}$ However, the resulting increase in radiation dose, particularly from increasing peak voltage, should be taken into consideration. ${ }^{5}$ Dual-energy CT techniques can be used to drastically reduce artifacts around implants ${ }^{6,7}$ while maintaining radiation exposure similar to standard single-energy protocol levels. ${ }^{8}$

MR imaging provides an excellent anatomical overview and detailed visualization of cement-implant and boneimplant interfaces and of the periarticular soft tissues such as pseudocapsule, tendons, and neurovascular structures.
Issue Theme Update in Hip Imaging; Guest Editor, Donna G. Blankenbaker, MD.
Copyright (c) 2013 by Thieme Medical Publishers, Inc., 333 Seventh Avenue, New York, NY 10001, USA. Tel: +1(212) 584-4662.
DOI http://dx.doi.org/ 10.1055/s-0033-1348098. ISSN 1089-7860. 
Owing to its superior soft tissue contrast, MR imaging is the preferred technique to assess synovitis, adverse local tissue reactions, and periarticular masses. ${ }^{9}$ During the June 2012 Orthopaedic and Rehabilitation Devices Advisory Panel meeting of the Food and Drug Administration, panel members recommended imaging using MRI with metal artifact reduction for the imaging of hip arthroplasty. ${ }^{10}$

Susceptibility differences around a hip arthroplasty result in altered precessional frequencies and locally accelerated dephasing of excited hydrogen, which is proportional to the field strength. This results in spatial misregistration, signal voids, and summation artifacts. Arthroplasty-related artifacts can be minimized by imaging at $1.5 \mathrm{~T}$ instead of $3 \mathrm{~T}$, using wide receiver bandwidth, thin slice thickness, a high number of excitations, and moderate echo times that preserve signalto-noise and contrast-to-noise ratios. Inversion recovery sequences provide homogeneous fat suppression compared with frequency selective fat saturation techniques. A 512 pixel readout matrix will improve the definition at the artifact interface even though it does not reduce susceptibility artifact. Dedicated metal artifact reduction techniques, such as multi-acquisition variable-resonance image combination $(\text { MAVRIC })^{11}$ and slice encoding for metal artifact correction (SEMAC), ${ }^{12}$ substantially reduce metal artifacts and improve the visualization of bone- and cement-arthroplasty interfaces and synovium (- Table $\mathbf{1}$ ).

\section{Hip Arthroplasty}

Total hip arthroplasties can be grouped by their bearing surfaces into metal femoral head on polyethylene acetabular liner (metal-on-polyethylene), ceramic head on polyethylene, ceramic head on ceramic liner, and metal-on-metal designs.

Optimal acetabular component position ensures stability, which depends on a complex relationship between acetabular abduction angle, acetabular anteversion, and femoral anteversion. ${ }^{13,14}$ The "safe zone" for cup positioning is $45^{\circ} \pm 10^{\circ}$ abduction and $20^{\circ} \pm 10^{\circ}$ anteversion. ${ }^{14}$ In general, $45^{\circ}$ of cup abduction, $15^{\circ}$ of cup anteversion, and $15^{\circ}$ femoral anteversion appear to provide high stability while preserving a wide joint range of motion. ${ }^{15}$ Femoral components should be placed in either mild valgus position so that the tip of the stem is located in the medial aspect of the medullary canal, or in a neutral position with the tip located centrally in the medullary canal.

Hip resurfacing arthroplasty consists of a noncemented acetabular component and a femoral component resembling a pegged metal cap that covers the femoral head. The femoral neck is preserved, which allows conversion to a total hip arthroplasty with a femoral shaft stem later in life. The femoral component should be placed in a $5-10^{\circ}$ valgus position relative to the femoral neck. ${ }^{16}$ Varus positioning or excessive valgus positioning can lead to notching of the femoral neck with resultant fracture or loosening. The recommended range of positioning of the acetabular component in metal-on-metal designs is 30 to $50^{\circ}$ abduction and 15 to $25^{\circ}$ of anteversion depending on the manufacturer. ${ }^{17,18}$

Implant fixation may be achieved through cementation of the component into bone or through cementless fixation. The goal of cementless fixation is the creation of a durable, biological, and mechanical link between the implant and the host bone. Cementless fixation is facilitated through the interdigitation of bone into coated implant surfaces ${ }^{19}$ (-Fig. 1).

On imaging, a well-fixed component demonstrates apposition of bone- or cement-implant interfaces without separation (-Figs. 1 and $\mathbf{2}$ ).

The presence of three or more of the following signs carries a positive predictive value of $96.9 \%$ for the presence of osseous integration of uncemented, porous-coated acetabular components: absence of linear radiolucencies along the bone-implant interface, presence of a superolateral and inferomedial buttress, medial stress shielding, and radial trabeculae. $^{20}$

\section{Complications}

Despite improvements in arthroplasty design, fixation techniques, and bearing materials and a resultant increase in survivorship, all implants eventually fail. Implant fixation and

Table 1 Imaging parameters of the hip at $1.5 \mathrm{~T}$ in the presence of metal

\begin{tabular}{|l|l|l|l|l|l|}
\hline Parameter & FSE axial & FSE sagittal & FSE coronal & Coronal MAVRIC & $\begin{array}{l}\text { Coronal inversion } \\
\text { recovery MAVRIC }\end{array}$ \\
\hline TR (ms) & $4500-5000$ & $5500-6500$ & $4500-5500$ & $4000-6000$ & $4000-6000$ \\
\hline TE (ms) & $24-34$ & $25-34$ & $25-34$ & $21-43$ & $21-43$ \\
\hline FOV (cm) & $17-26$ & $18-26$ & $18-26$ & $26-39$ & $26-39$ \\
\hline Matrix & $512 \times 256-320$ & $512 \times 320-352$ & $512 \times 320-384$ & $512 \times 256-384$ & $256 \times 192$ \\
\hline $\begin{array}{l}\text { Slice thickness } \\
(\mathrm{mm}) / \text { gap }\end{array}$ & $4 / 0$ & $3-4 / 0$ & $4 / 0$ & $3-4.5 / 0$ & $3-4.5 / 0$ \\
\hline RBW (kHz/px) & 488.3 & 488.3 & 488.3 & 488.3 & 488.4 \\
\hline NEX & $4-5$ & $4-5$ & $4-5$ & 0.5 per frequency bin & 0.5 per frequency bin \\
\hline
\end{tabular}

Abbreviations: FSE, fast spin echo; MAVRIC, multi-acquisition variable-resonance image combination; FOV, field of view; RBW, receiver bandwidth; NEX, number of excitations. 

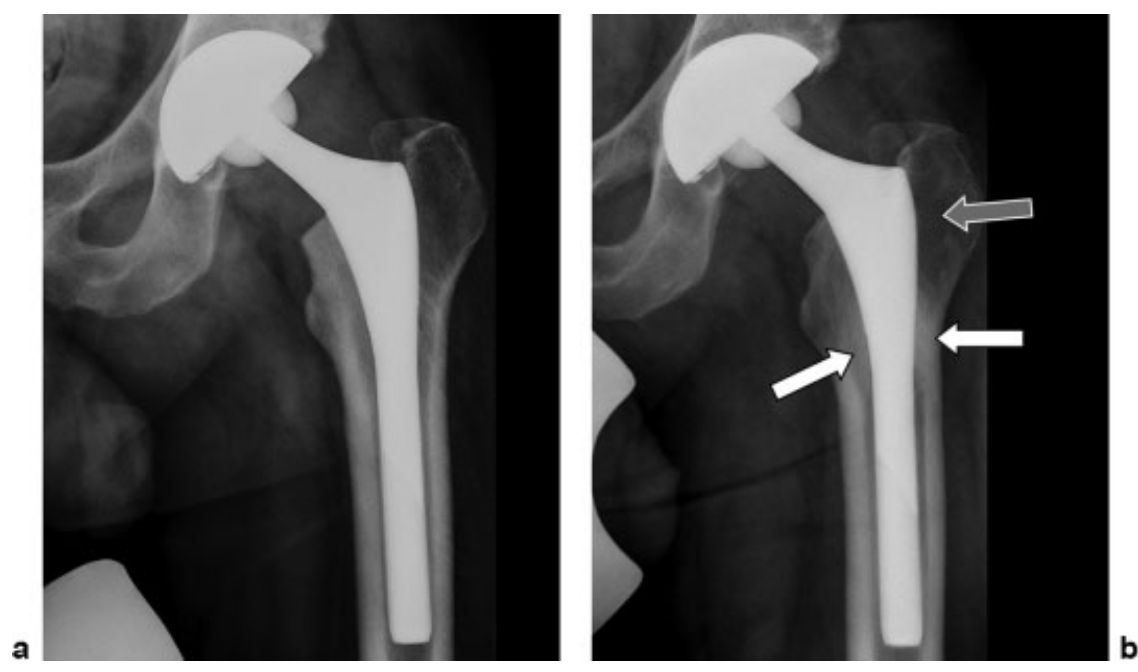

Figure 1 A 44-year-old man with ceramic-on-polyethylene left hip arthroplasty. (a) Anteroposterior radiograph demonstrates well-fixed acetabular and femoral components without radiolucencies along the bone-implant interfaces. (b) Anteroposterior radiograph obtained 3 years later demonstrates interval development of bony ingrowth around the proximal stem indicated by circumferential sclerosis extending to the implant surface (white arrows). Note decreasing osseous density in the greater trochanter as a result of stress shielding (gray arrow).

joint stability are key factors for longevity. Common modes of failure are loosening, infection, and periprosthetic fractures. ${ }^{21}$ Additional complications include synovitis and adverse local tissue reactions due to wear, as well as tendinopathy, heterotopic ossification, and neuropathy.

\section{Mechanical Loosening and Membrane Formation}

Loosening is defined as the complete loss of fixation of an implant at surgery. Mechanical or aseptic loosening is inferred if an infection work-up is negative and no wearinduced synovitis is present. Mechanical stress is thought to promote migration of synoviocytes into bone-implant and bone-cement interfaces, ${ }^{22}$ which leads to the formation of a "fibrous" or "synovial-like" membrane and to the release of osteoclast-stimulating cytokines. ${ }^{23}$ Up to $60 \%$ of arthroplasties are revised due to loosening. ${ }^{21}$ Membrane formation and bone resorption along interfaces can precede loosening. On radiographs and $\mathrm{CT}$, linear radiolucencies are seen along the interface, and on MRI a smooth intermediate signal intensity layer is visible (-Fig. $\mathbf{3}$ ).

Loosening may only be diagnosed with certainty on imaging if there is implant displacement, progressive subsidence, contrast material surrounding an implant entirely at arthrography, or a 2 degrees or more difference of version angles on CT images obtained in maximum external and internal rotation. ${ }^{24}$

Bone resorption and osteolysis around a femoral component are described with reference to the Gruen zones ${ }^{25}$ (-Fig. 4) and around the acetabular component bone using the zones of DeLee and Charnley on radiographs ${ }^{26}$ (- Fig. 4). Similar periacetabular abnormalities on CT and MRI are described with respect to the acetabular walls and pelvic columns.

Mild bone resorption in Gruen zone 1 and decreased radiodensity of the greater trochanter are common findings, which occur secondary to "stress shielding" (-Fig. 1). Stress
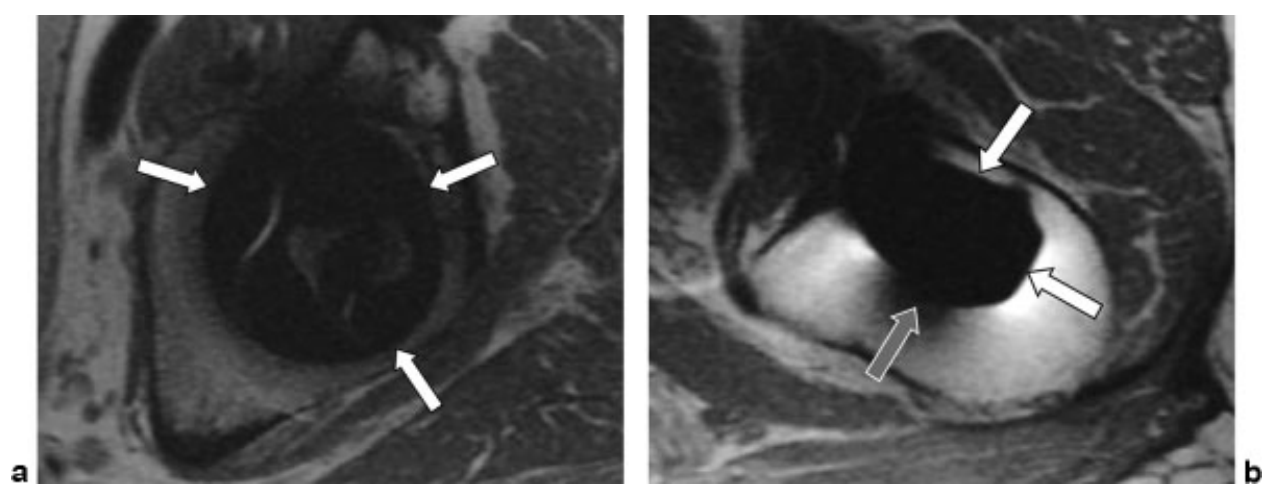

Figure 2 A 48-year-old man with metal-on-metal left hip arthroplasty. (a) Axial fast spin-echo MR image demonstrates a well-fixed acetabular component with cancellous bone extending to the implant surface (arrows) and no interposition of abnormal signal intensity. (b) Axial fast spinecho MR image demonstrates a well-fixed femoral component with cancellous bone (white arrows) and a focus of sclerosis (gray arrow) extending to the implant surface. 

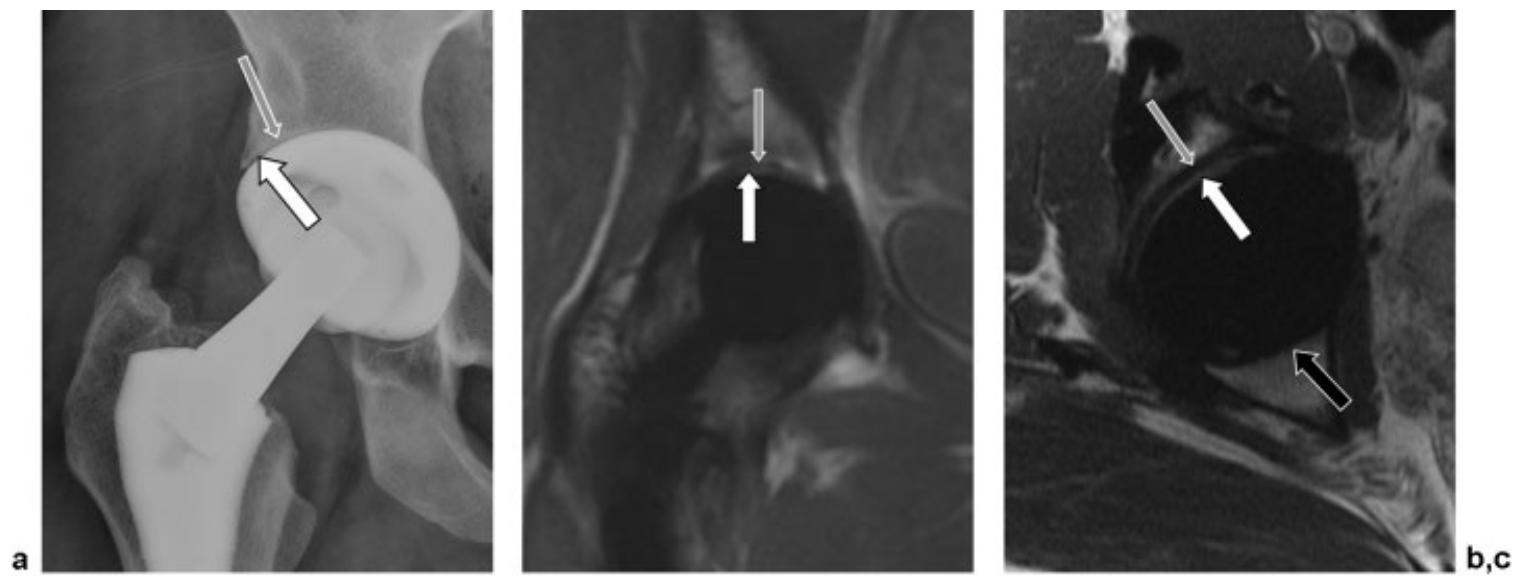

Figure 3 Fibrous membrane formation in a 45 year old woman with a ceramic-on-ceramic right hip arthroplasty. (a) Anteroposterior radiograph demonstrates a thin radiolucency (white arrow) with a subjacent rim of sclerosis (gray arrow) in zone 1. (b) Coronal multi-acquisition variableresonance image combination and (c) axial intermediate-weighted fast spin-echo MR images demonstrate fibrous membrane formation over the acetabular roof (b) and anterior wall (c) with an intermediate to high signal intensity layer (white arrows) situated between the component and a low signal intensity rim of sclerosis (gray arrows). Because there is osseous integration over the posterior wall (c, black arrow), the component is not loose.

shielding describes a process by which the implant shifts physiologic load away from the greater trochanter. ${ }^{27}$ Calcar resorption of the femur may also occur due to stress shielding. ${ }^{28}$

As a general rule, bone resorption $<1 \mathrm{~mm}$ is considered clinically insignificant, 1 to $2 \mathrm{~mm}$ is considered to represent membrane formation, and $>2 \mathrm{~mm}$ indicates localized loosening. Although the significance of membrane formation is unclear, it may represent a metastable state that warrants imaging surveillance.

In uncemented acetabular components, ${ }^{29}$ the presence of radiolucencies $>2 \mathrm{~mm}$ in thickness in zone 3 indicate instability, radiolucencies $<2 \mathrm{~mm}$ in zone 3 indicate stable fibrous membrane formation, and linear radiolucencies of 1 to $2 \mathrm{~mm}$ are permissible in zone 1 or 2 , if solid integration is present in zone 3.
In uncemented femoral components, ${ }^{30}$ predictors of unstable fibrous integration (loosening) are component migration, progressive radiolucencies, nonparallel or divergent radiolucencies, and bony hypertrophy at the tip of the femoral component (pedestal formation). The presence of spot-weld densities at the end of the porous coated surface, absence of radiolucencies next to the porous coating, and calcar resorption secondary to stress shielding indicate stable integration. Signs suggestive of loosening are shedding of the coated surface, endosteal scalloping, and bone resorption that appears after or progresses $>2$ years after arthroplasty replacement.

In cemented femoral components, ${ }^{31}$ radiolucencies involving $>50 \%$ but $<100 \%$ of the cement-bone interface on one or more radiographs indicate possible loosening, radiolucencies surrounding the entire component $(100 \%$ of the
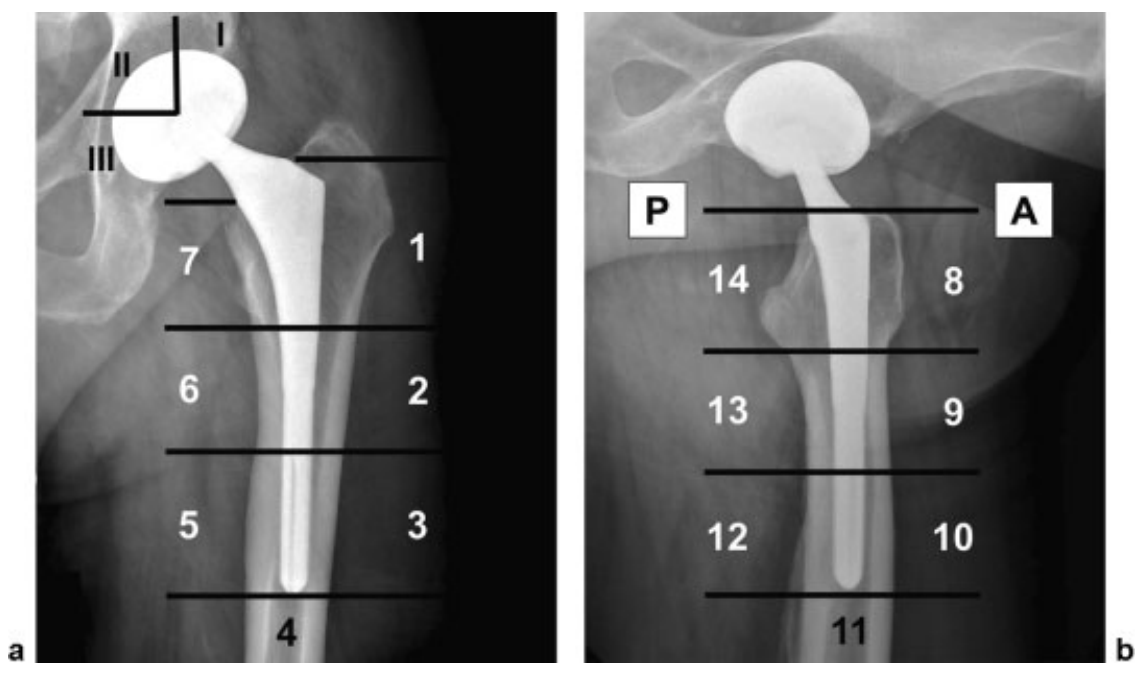

Figure 4 Acetabular and femoral component zones. (a) Anteroposterior radiograph of a right hip arthroplasty demonstrates the acetabular component zones after De Lee and Charnley (I-III) and the femoral Gruen zones 1 to 7. (b) Lateral radiograph of a right hip arthroplasty demonstrates the femoral Gruen zones 8 to 14 . A, anterior; P, posterior. 

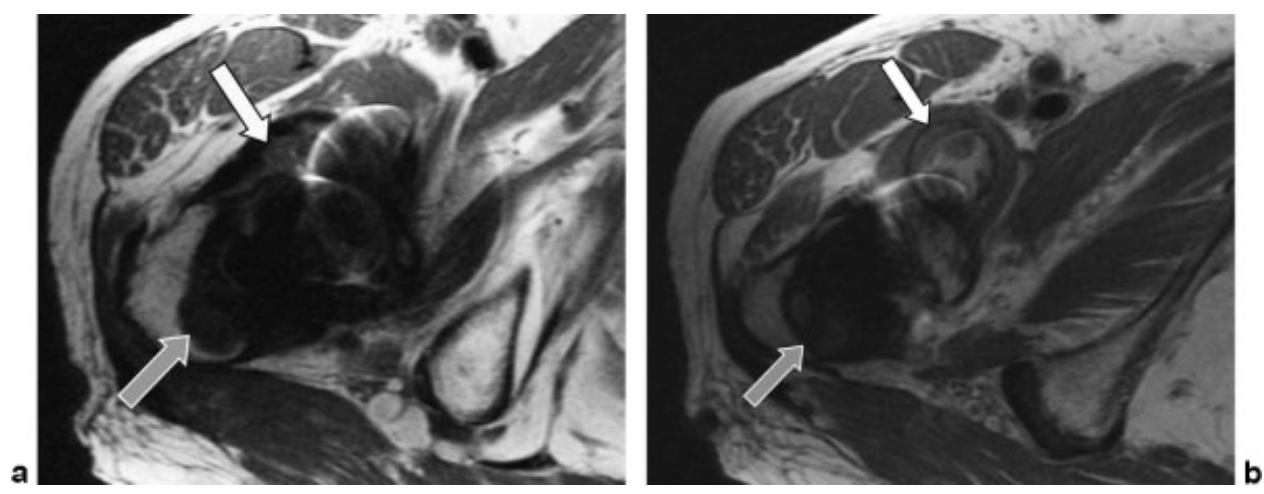

Figure 5 Polyethylene wear-induced synovitis in an 81-year-old man with metal-on-polyethylene right hip arthroplasty. (a, b) Axial fast spin-echo MR images demonstrate expansion of the pseudocapsule by intermediate signal intensity debris (a, white arrow) with decompression into the iliopsoas bursa (b, white arrow), which demonstrates a thickened wall, intermediate signal intensity debris, and high signal intensity fluid. Note the partially visualized low signal intensity osteolysis in Gruen zone 1 and 14 (a and b, gray arrows).

cement-bone interface) on one or more radiographs that were not present on immediate postoperative radiographs indicate probable loosening, and discernible component or cement migration indicates definite loosening. 1 to $2 \mathrm{~mm}$ of subsidence may occur during the first year after implantation; however, subsidence $>5 \mathrm{~mm}$ is a sign of loosening. ${ }^{32}$ Cement fracture and shift of stem position also indicate loosening.

\section{Polyethylene Wear}

All components of a hip arthroplasty undergo wear, leading to shedding of debris. Ultra high molecular weight polyethylene debris has the highest inflammatory profile, potentially inciting a histiocytic reaction that leads to a cytokine-mediated upregulation of osteoclast function and subsequent osteolysis. ${ }^{33}$ Although there is no threshold for polymeric induced osteolysis, polyethylene components should ideally have a linear wear rate $<0.1$ to $0.2 \mathrm{~mm} / \mathrm{year}^{34,35}$ Newer, highly cross-linked polyethylenes have better wear profiles $^{35,36}$ and retain versatility and third-body wear resistance, but they may still incite osteolysis.

On radiographs, osteolysis is suggested by geographic radiolucencies $>2 \mathrm{~mm}$ in thickness. ${ }^{37}$ However, radiographs are less sensitive for the depiction of osteolysis ${ }^{38}$ when compared with CT and MRI, with quoted sensitivities for the detection of osteolysis of $52 \%$ for radiographs, $75 \%$ for CT, and $95 \%$ for MRI. ${ }^{39}$

On MRI, polyethylene wear-induced synovitis often presents as distention of the pseudocapsule by low to intermediate signal intensity debris (-Fig. 5). Increased intraarticular pressures can result in extension of wear debris into the iliopsoas and subiliacus bursae ( $\mathbf{F i g . 5}$ ). Osteolysis due to polymeric debris is typically bulky and geographic in appearance and manifests as intermediate to high signal intensity material replacing the normal bone and marrow signal surrounding the implant (-Fig. 6). Large extra-articular soft tissue deposits may be seen that resemble the pseudotumors seen in adverse local tissue reactions. Knowledge of the type of arthroplasty, the timing of arthroplasty placement, and associated soft tissue findings helps to distinguish these pathologically and histologically distinct entities. For example, synovitis in a recently placed metal-on-polyethylene arthroplasty with a modular neck-stem junction is more likely to represent an adverse tissue reaction due to hypersensitivity than a polymeric wear-induced synovitis due to macrophage activation because polyethylene synovitis is a
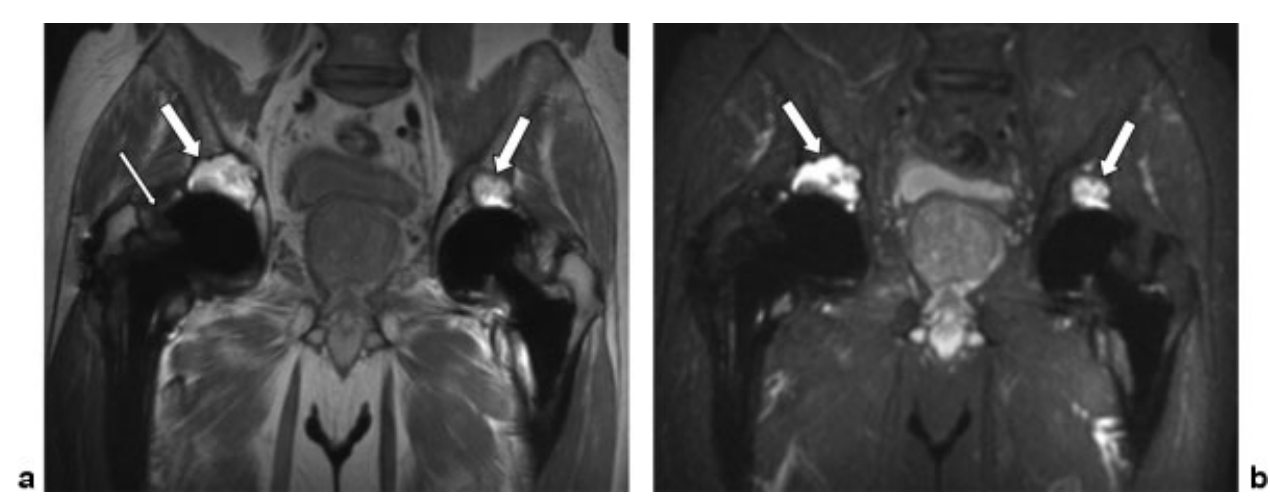

Figure 6 Polyethylene wear-induced osteolysis of the acetabulum bilaterally in a 73-year-old woman with metal-on-polyethylene hip arthroplasties. (a) Coronal intermediate-weighted and (b) inversion recovery multi-acquisition variable-resonance image combination MR images demonstrate geographic osteolysis bilaterally (thick white arrows) and distention of the pseudocapsule by low and intermediate signal intensity synovitis and debris (thin white arrow). 
slowly progressing process. Accurate characterization of the signal within the expanded hip pseudocapsule with the use of metal artifact reduction sequences may help in the differentiation of polymeric reaction, infection, and adverse local soft tissue reactions.

\section{Adverse Local Soft Tissue Reactions}

The term adverse local soft tissue reaction (ALTR) encompasses various reactions to the presence of metal debris, metal ions, and corrosion products in metal-on-metal or modular arthroplasty systems. ${ }^{40}$ Types of ALTR include hypersensitivity reactions to metal products typically in the setting of low wear, metallosis without hypersensitivity in the setting of high wear, and a combination of both. ${ }^{41}$ The terms pseudotumor and adverse reaction to metal debris have been used synonymously with ALTR. ${ }^{41}$

Adverse local soft tissue reactions can occur in both well-positioned and malpositioned metal-on-metal arthroplasty systems because there are several ways in which metal products can be shed into the periprosthetic soft tissues. For example, accelerated wear of bearing surfaces may occur when the arthroplasty alignment is outside the safe zone of optimal component positioning. In addition, fretting and corrosion may occur at the head-neck junction (also known as the trunnion) and neck-stem junction of modular systems, which may account for the presence of an ALTR with metal-on-polyethylene bearing surfaces. ${ }^{42}$ Corrosion is an electrochemical process that may occur anywhere along the arthroplasty surface but is particularly problematic at the neck-stem junction and at the trunnion, contributing to the presence of metal ions locally and in the bloodstream as well as corrosion products like chromium orthophosphate in the local tissues. ${ }^{42}$

Aseptic lymphocytic vasculitis associated lesion (ALVAL) is the histologic appearance of hypersensitivity to metal. These reactions are characterized microscopically by the presence of lymphocytes, histiocytes, intracytoplasmic wear debris, and necrosis in varying proportions. A 10-point histologic scoring system based on the integrity of the synovial lining, the degree and type of inflammatory infiltrate, and tissue organization indicates the presence of ALVAL and correlates with the degree of tissue damage. ${ }^{43}$ An ALVAL score of $\geq 5$ is consistent with a diagnosis of moderate to severe hypersensitivity. ${ }^{44}$ Hypersensitivity reactions typically occur at low wear rates and are associated with high ALVAL scores. By contrast, metallosis is seen in the setting of high wear rates and low ALVAL scores.

Although it is believed to represent a type IV hypersensitivity reaction, the exact causative factors involved in ALVAL are currently unknown. Serum ion levels alone have a $63 \%$ sensitivity and $86 \%$ specificity for predicting a hypersensitivity reaction to metal ions, suggesting an idiosyncratic patientdependent sensitivity to the development of an adverse tissue reaction in response to elevated serum and soft tissue ion levels ${ }^{45}$ In addition to patient-specific factors, the host response may also be affected by the duration of exposure and by the size, type, and concentration of metal debris, metal ions, and corrosion products. ${ }^{42}$

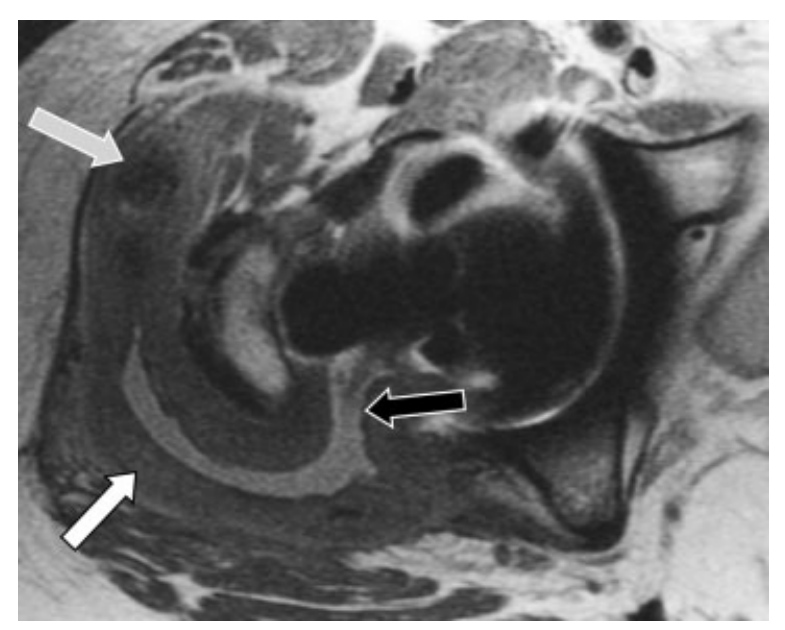

Figure 7 Adverse local tissue reaction in a 54-year- old woman with metal-on-metal hip arthroplasty. Axial fast spin-echo MR image demonstrates focal disruption of the lateral margin of the posterior pseudocapsule (black arrow) allowing for synovial debris and synovitis to decompress into the posterior soft tissue envelope and thereby into the greater trochanteric bursa. The markedly thickened rind of the fluid collection (white arrow) is consistent with an aggressive adverse local tissue reaction and suggests the presence of a hypersensitivity reaction to metal ions and aseptic lymphocytic vasculitis associated lesion. Foci of diminished signal intensity anterior to the greater trochanter (gray arrow) suggest the concomitant presence of metallosis.

MRI with the use of optimized imaging parameters and metal artifact reduction techniques has a high predictive value for diagnosing patients with an adverse local soft tissue reaction. ${ }^{44}$ Synovial thickening and a large volume of synovitis are two variables of high importance in predicting hypersensitivity associated with a high ALVAL score (-Fig. 7). Other pertinent features on MRI include a mixed-type synovitis, consisting of synovial fluid and solid synovial debris; dehiscence of the pseudocapsule; soft tissue edema; osteolysis; abductor disruption; neurovascular compromise; and local adenopathy. ${ }^{9,11}$ Pseudocapsular dehiscence is most commonly seen at the posterolateral attachment of the pseudocapsule and allows for fluid and debris to decompress into the greater trochanteric bursa (-Fig. 7). This is particularly problematic when the adverse tissue reaction comes into contact with the hip abductor tendon insertions, which can then rupture. Posterior extension may also compromise the sciatic and posterior femoral cutaneous nerves. Anterior pseudocapsular dehiscence is also common, in which case the adverse tissue reaction may extend into the iliopsoas and subiliacus bursae, potentially compromising the iliopsoas tendon and femoral neurovascular bundle.

By contrast, metallosis is associated with lower signal intensity material and a relative lack of soft tissue disruption. Metal products can either coat the synovium or form confluent intra-articular and intraosseous deposits similar in morphology but lower in signal intensity than polymeric debris. 


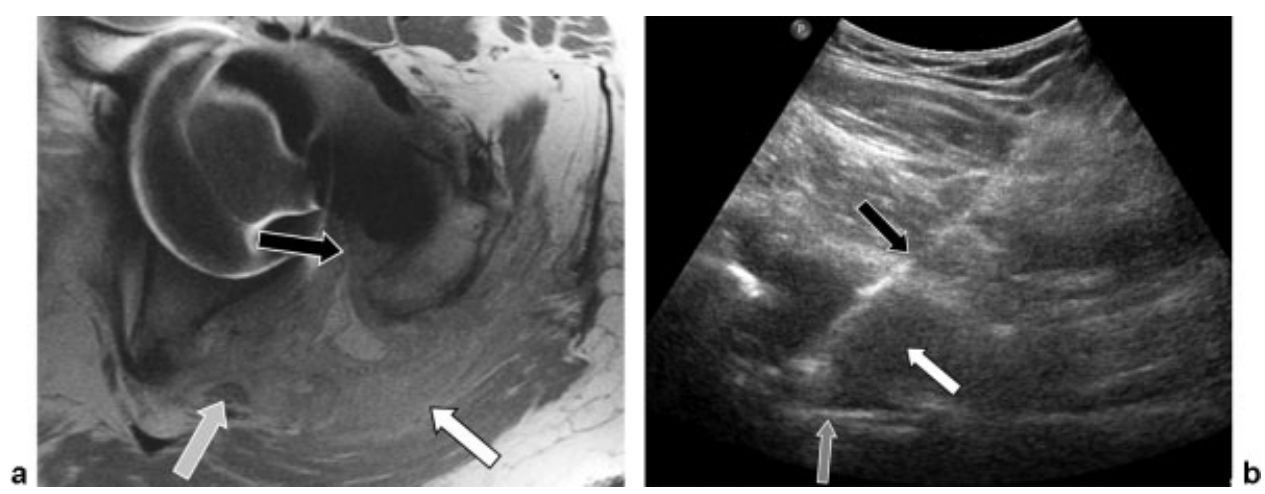

Figure 8 Adverse local tissue reaction in a 62-year-old man with left metal-on-metal hip arthroplasty. (a) Axial fast spin-echo MR image demonstrates focal disruption of the lateral margin of the posterior pseudocapsule (black arrow) allowing for synovitis to decompress into the posterior soft tissue envelope. This aggressive adverse local tissue reaction (white arrow) abuts the left sciatic nerve (gray arrow) with effacement of fat planes. The differential diagnosis includes infection, which was ruled out by joint aspiration. Aseptic lymphocytic vasculitis associated lesion was confirmed histologically through synovial biopsy. (b) Ultrasound-guided synovial biopsy with biopsy needle (black arrow) in the thickened hypoechoic synovium (white arrow). Gray arrow points to the neck of the prosthesis.

When the diagnosis of an ALTR is suspected on MRI, ultrasound is useful for guiding joint aspirations and synovial biopsies (-Fig. 8).

\section{Infection}

The diagnosis of periprosthetic infection is difficult, often requiring multimodality assessment and the use of imageguided aspiration for confirmation. Serological tests including white cell count, erythrocyte sedimentation rate (ESR), and Creactive protein (CRP) have a good sensitivity but low specificity. ${ }^{46}$ The ESR and CRP normally rise after joint arthroplasty with CRP falling to normal levels after 3 weeks. ESR can take longer than CRP to return to normal levels, remaining elevated for up to 6 weeks.

Radiographs are insensitive for the detection of implant infection. Periosteal reaction and osteolysis can be seen in the setting of infection but are not specific because similar findings can be seen in aseptic loosening and stress reaction.

Technetium 99m-methylene diphosphonate bone scans are nonspecific and may remain positive for years around ingrowth components, but high diagnostic accuracy has been reported with combined white blood cell and bone marrow scintigraphy. 47

The MR imaging features of infection include joint effusion, extracapsular/synovial/osseous edema and enhancement, extracapsular collections, bone destruction, and reactive lymphadenopathy. ${ }^{48}$ In a study of infected knee arthroplasties, a "lamellated" hyperintense appearance of the synovium (defined as thickened synovium composed of multiple layers) had a high positive predictive value for infection as an isolated finding ${ }^{49}$ (- Fig. 9). The positive predictive value is increased when this is combined with secondary findings such as extracapsular soft tissue edema, local lymphadenopathy, and extracapsular collections (-Fig. 9). Features of osteomyelitis and aggressive bone destruction should be sought, but their absence does not exclude infection. A similar set of findings can be expected in the hip, although these findings have not yet been systemati- cally investigated in joints other than the knee. Image-guided joint aspiration and lavage are ultimately required to exclude potential periprosthetic infection (-Fig. 9).

\section{Periprosthetic Fracture}

Periprosthetic fractures occur with overall incidence of 0.1 to $18 \%{ }^{50}$ Most periprosthetic fractures occur around the femoral component. Etiologies of postoperative fractures include periprosthetic bone resorption, osteolysis, loosening, and occasionally trauma. Femoral component varus position and previously treated fractures are additional risk factors.

The Vancouver classification of periprosthetic fractures is based on fracture location, implant stability, and bone stock, all of which affect prognosis and guide management. ${ }^{51}$ This classification system recognizes three femoral zones, which are the trochanteric region ( $A, 4 \%$ incidence), diaphysis around the implant (B, 86.7\%), and the femur distal to the tip of the femoral component ( $C, 9.3 \%)$. Type A fractures are considered stable if only minimally displaced $(<2 \mathrm{~cm}) .{ }^{52}$ Type B1 describes a periprosthetic fracture with well-fixed femoral component and good bone stock, which can be treated with open reduction internal fixation techniques. In both type B2 and B3, the implant is loose, with the addition of poor bone stock in type B3. Displacement of the femoral component with respect to the femur indicates loosening, whereas circumferential radiolucencies at the bone-implant or bone-cement interface may require manual intraoperative confirmation. B2 and B3 fractures require revision hip arthroplasty. Type $C$ fractures are managed similar to distal femur fractures. The degree of displacement of a periprosthetic fracture is important because conservative management of a nondisplaced or minimally displaced fracture can be attempted with protected weightbearing. In cases of nontraumatic periprosthetic fracture, infection work-up should be initiated.

CT and MRI represent powerful problem-solving tools in radiographically indeterminate cases. Optimized MR imaging of hip arthroplasty is the most accurate modality because of 

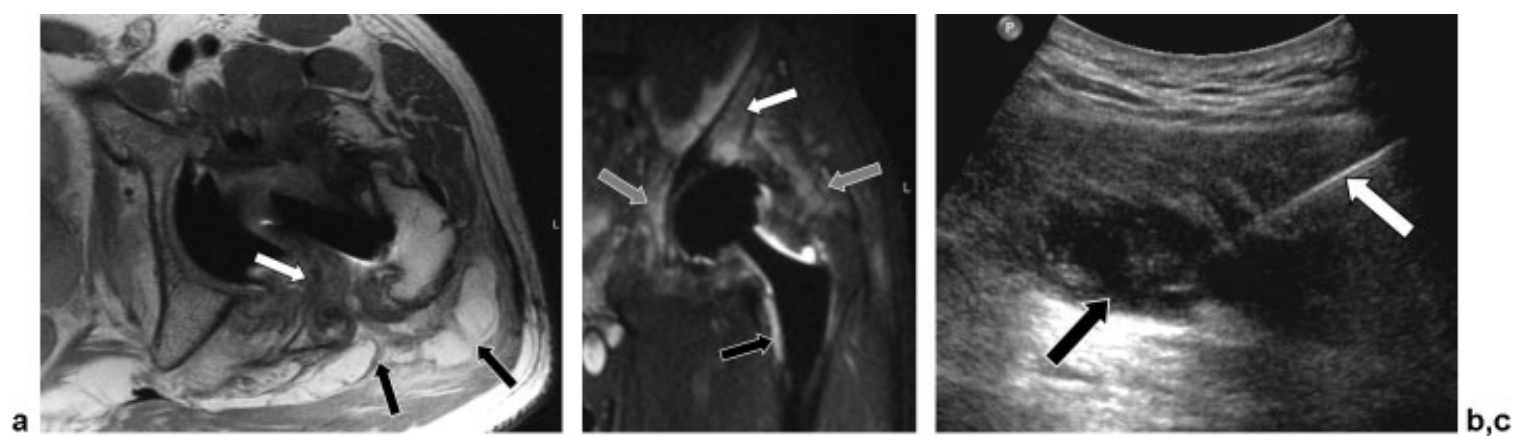

Figure 9 Infected ceramic-on-ceramic hip arthroplasty in a 38-year-old man. (a) Axial fast spin-echo MR image demonstrates a thickened and lamellated synovium (white arrow) with focal disruption of the lateral margin of the posterior pseudocapsule and formation of a loculated highintensity signal collection in the posterior soft tissue envelope (black arrows). (b) Coronal inversion recovery multi-acquisition variable-resonance image combination MR image demonstrates bone marrow edema pattern in the ilium (white arrow) and Gruen zone 7 (black arrow) as well as regional soft tissue edema (gray arrows). (c) Ultrasound-guided aspiration with needle (white arrow) in the posterolateral, loculated heterogeneous collection (black arrow).

the ability to demonstrate stress reactions (-Fig. 10) and subtle and nondisplaced fractures (- Fig. 11). MR imaging signs include a localized bone marrow edema pattern, periosteal reaction, and hypointense fracture lines that may be complete or incomplete ${ }^{53}$ (-Fig. 11).

\section{Heterotopic Ossification}

Heterotopic bone formation is defined as the formation of mature lamellar bone with trabeculae in nonosseous tissues. ${ }^{54}$ Mature heterotopic bone is traditionally diagnosed on anteroposterior radiographs and characterized by welldemarcated cortices and a trabecular pattern. The Brooker classification of "ectopic ossification" has been widely adopted. ${ }^{55}$ Class 1 describes islands of heterotopic ossification within periarticular soft tissues. Class 2 describes approximating spurs of heterotopic ossification originating from the periacetabular region and femur, leaving at least
$1 \mathrm{~cm}$ between approximating interfaces. Class 3 describes a similar constellation as class 2 , but with $<1 \mathrm{~cm}$ of a gap. Class 4 describes ankylosing heterotopic ossification between periacetabular bone and femur. Class 3 , which is typically associated with a meaningful loss of joint motion, may be misinterpreted as class 4 on anteroposterior radiographs because of superimposition. In such cases, $\mathrm{CT}$ is useful for accurate characterization. ${ }^{56}$ On MR imaging, mature heterotopic ossification has the appearance of cancellous bone with a thin hypointense cortex and fatty marrow (-Fig. 11), which can be suppressed or saturated. Because of the similarity of the MR signal to juxta-articular fat tissue, which may be abundantly present in cases of advanced fatty muscle atrophy, heterotopic ossification may be difficult to detect on MR images. Heterotopic ossification or extruded cement may exceed mass effect on the abductor and iliopsoas tendons and may impinge on nerves.
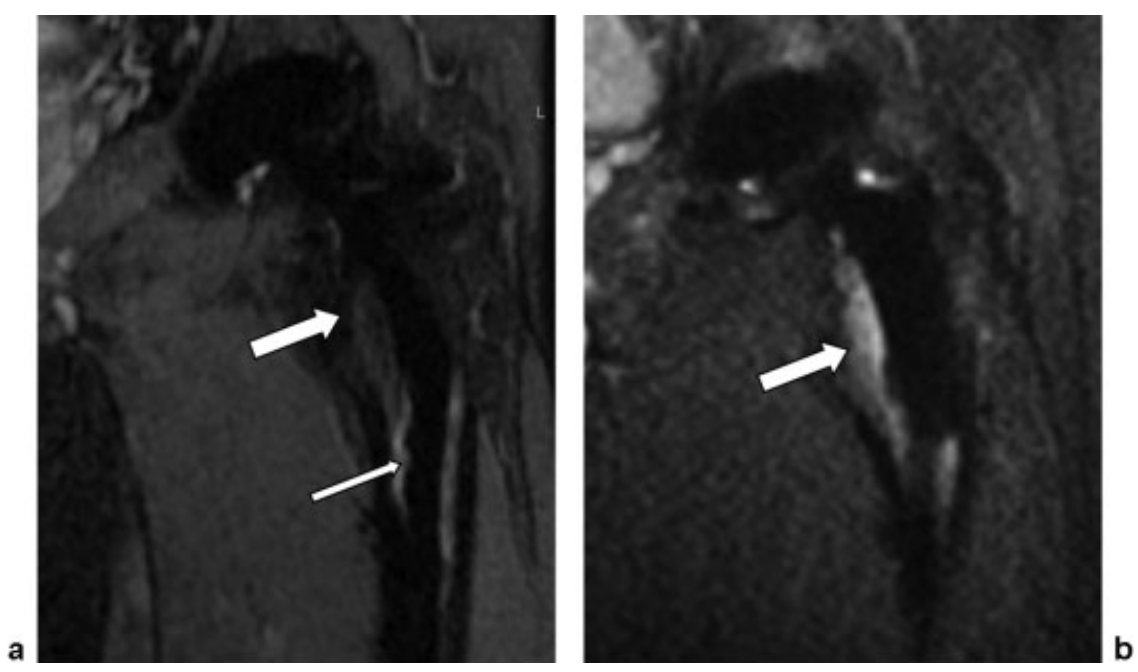

Figure 10 Osseous stress reaction in a 52-year-old man with a ceramic-on-polyethylene hip arthroplasty. (a) Coronal inversion recovery multi-acquisition variable-resonance image combination (MAVRIC) MR image obtained 6 months following implantation demonstrates normal bone marrow signal intensity along the proximal femoral (thick white arrow) bone-implant interface. Note mild edema pattern following reaming (thin white arrow). (b) Coronal inversion recovery MAVRIC MR image obtained 3 years following implantation demonstrates a new bone marrow edema pattern along the bone-implant interface of Gruen zones 7 (arrow) and 1 of the femoral component, indicative of a stress reaction. There was no fracture line. 

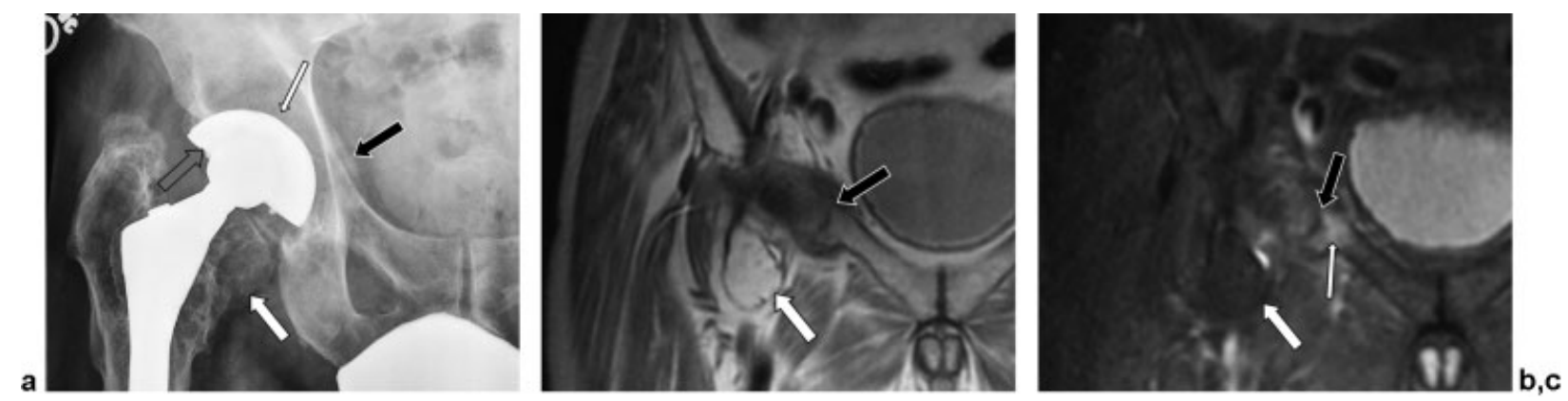

Figure 11 Superior ramus fracture in a 44-year-old man with metal-on-polyethylene hip arthroplasty. (a) Anteroposterior radiograph demonstrates a subtle periosteal reaction along the iliopectineal line (black arrow). Note heterotopic ossification medial to the femoral neck (white arrow), eccentric liner wear (gray arrow), and geographic periacetabular radiolucency (thin white arrow). (b) Coronal intermediateweighted multi-acquisition variable-resonance image combination (MAVRIC) MR image demonstrates a fracture line through the superior ramus (black arrow). Note the high signal intensity focus of heterotopic ossification (white arrow). (c) Coronal inversion recovery MAVRIC MR image demonstrates edema pattern (thin white arrow) adjacent to the fracture line (black arrow). Note suppression of the fatty marrow of the focus of mature heterotopic ossification (thick white arrow).

\section{Tendinopathies}

Disorders of the peritrochanteric region of the hip including greater trochanteric bursitis and tears of the gluteus medius and minimus are important causes of morbidity following arthroplasty. Patients presenting with lateral hip pain are initially evaluated with radiographs to assess for the presence of periprosthetic fractures, avulsions of the greater trochanter, and heterotopic ossification. Radiographs in the setting of abductor tears are usually unremarkable, apart from the occasional identification of enthesopathic bony irregularity. ${ }^{57}$ On ultrasound, tendon tears are seen as focal areas of hypoechogenicity disrupting the normal fibrillary architecture of the tendon, with or without abnormal tendon thickening. ${ }^{58}$ Connell et al ${ }^{59}$ reported a sensitivity of $93 \%$ and specificity of $95 \%$ for diagnosing tendon tears on ultrasound, also noting associated irregularity of the greater trochanter in approximately half of their cases. Ultrasound is also useful to guide greater trochanteric bursal, iliopsoas bursal, and peritendinous injections.

MRI provides the most information about the status of periprosthetic tissues, allowing for multiplanar assessment with high soft tissue contrast. Tendinosis is seen as thickening and increased signal of the tendon. Partial-thickness tears are seen as defects with altered signal intensity, with or without tendon retraction, and they may be associated with peritendinous soft tissue edema in the acute setting. ${ }^{57}$ The hip abductors may become tendinotic or tear as a result of primary age-related degeneration, trauma, or overuse (-Fig. 12). Chronic high-grade or complete tears of the hip abductors can result in extensive scarring leading to an appearance of "scar-in-continuity" at the greater trochanter. The gluteus medius and minimus tendons may be scarred to one another or to the hip pseudocapsule or the overlying iliotibial band. Muscle atrophy due to damage to the superior
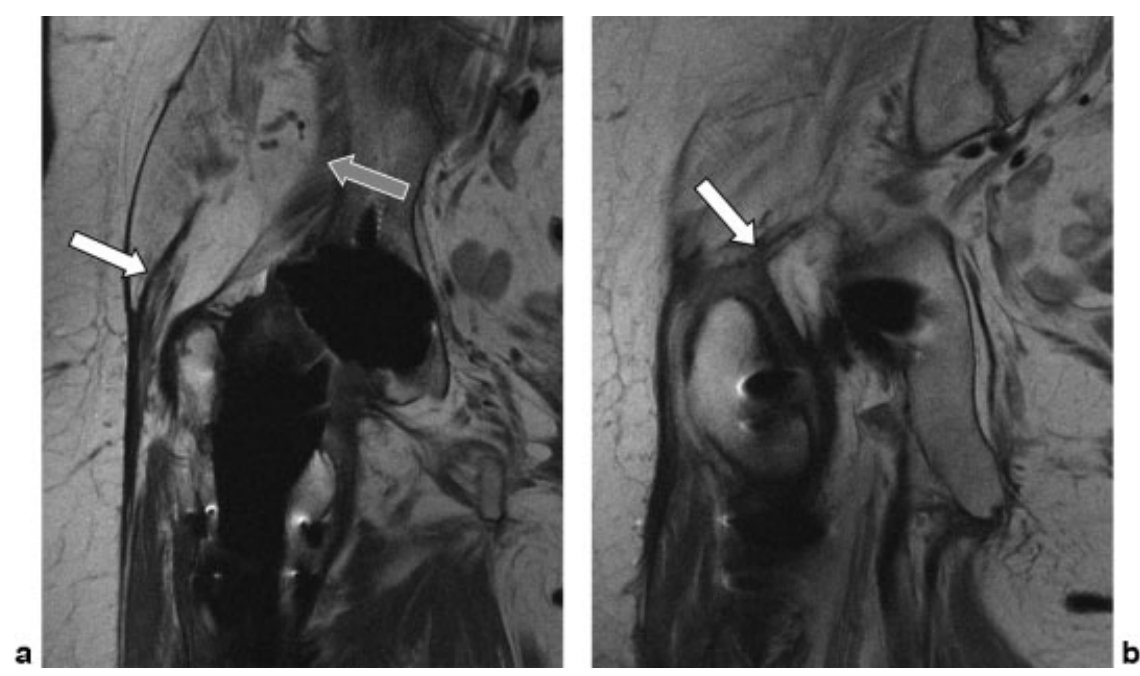

Figure 12 Abductor tendinopathy in a 77-year- old man with metal-on-polyethylene revision hip arthroplasty. (a) Coronal intermediate-weighted fast spin-echo MR image demonstrates chronic longitudinal stripping of the anterolateral footprint of the gluteus medius tendon (white arrow) with muscle atrophy. The gluteus minimus tendon is intact but the muscle is atrophic (gray arrow). (b) Coronal intermediate-weighted fast spinecho MR image demonstrates a partial tear of the posterior gluteus medius footprint with partial retraction (arrow). 

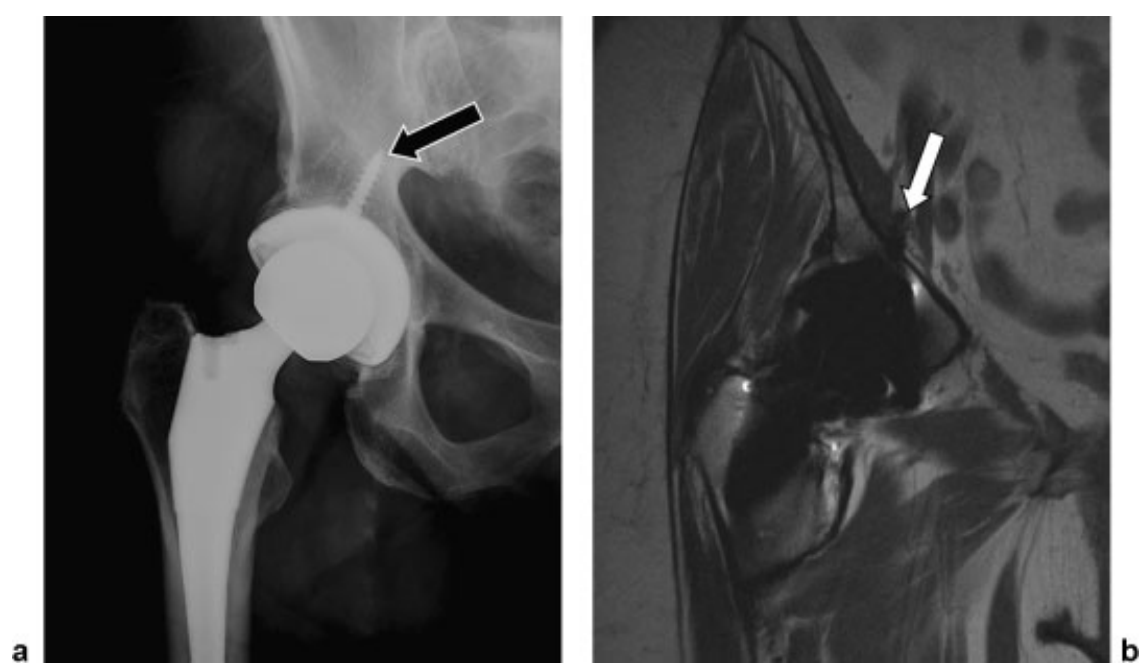

Figure 13 Iliopsoas impingement by an acetabular screw in a 71-year-old woman with metal-on-polyethylene hip arthroplasty. (a) Anteroposterior radiograph demonstrates an acetabular screw with apparent intraosseous location of the screw tip (arrow). (b) Coronal intermediate-weighted fast spinecho MR image demonstrates cortical penetration of the screw with the tip located at the iliacus muscle tendon junction (arrow).

gluteal nerve rather than due to tendon tear represents an important differential diagnosis in patients with limping after arthroplasty. ${ }^{60}$ Fatty atrophy of the anterior aspect of the gluteus minimus is a common finding in asymptomatic patients, analogous to subselective denervation of the teres minor in the shoulder. Defects of the abductor tendons and fatty atrophy of the posterior part of the gluteus minimus and the gluteus medius muscles are uncommon in asymptomatic patients and are therefore likely to be clinically relevant. ${ }^{60}$ Heterotopic ossification may also impinge on the hip abductors resulting in secondary deformity and tendon tears. Occasionally malposition of implants, cement, or aberrant placement of fixation screws may also impinge on tendons and bursae (-Fig. 13).

\section{Nerve Damage}

Nerve injury occurs in $~ 1$ to $2 \%$ of arthroplasties and is more common in cases of revision or acetabular reconstruction in the setting of underlying dysplasia. ${ }^{61}$ The sciatic nerve is the most commonly injured nerve during hip arthroplasty due to its relatively fixed position between the greater sciatic notch and the fibular head, predisposing it to stretch injury. Injuries to the superior gluteal nerve, lateral femoral cutaneous nerve, femoral nerve, and obturator nerves are less common but occur. Nerve injury can occur at the time of surgery due to direct nerve damage or transection, nerve stretch, or transient ischemia due to direct compression or vascular injury. Injury to nerves may also occur following dislocation events, postoperative hematoma, tendon avulsions, heterotopic ossification, or in the setting of adverse local soft tissue reactions (-Fig. 8). Malpositioned or displaced arthroplasty components may directly impinge on adjacent neurovascular structures. Marked distension of bursae around the hip may also displace or compress the sciatic and femoral nerves. MR imaging signs of nerve abnormality include intraneural or epineural high signal, loss of fascicular architecture, and neuroma formation.

\section{References}

1 Mancuso CA, Salvati EA, Johanson NA, Peterson MG, Charlson ME. Patients' expectations and satisfaction with total hip arthroplasty. J Arthroplasty 1997;12(4):387-396

2 Brown TE, Larson B, Shen F, Moskal JT. Thigh pain after cementless total hip arthroplasty: evaluation and management. J Am Acad Orthop Surg 2002;10(6):385-392

3 Miller TT. Sonography of joint replacements. Semin Musculoskelet Radiol 2006;10(1):79-85

4 Lee MJ, Kim S, Lee SA, et al. Overcoming artifacts from metallic orthopedic implants at high-field-strength MR imaging and multidetector CT. Radiographics 2007;27(3):791-803

5 Fritz J, Fishman EK, Corl F, Carrino JA, Weber KL, Fayad LM. Imaging of limb salvage surgery. AJR Am J Roentgenol 2012;198(3): 647-660

6 Bamberg F, Dierks A, Nikolaou K, Reiser MF, Becker CR, Johnson TR. Metal artifact reduction by dual energy computed tomography using monoenergetic extrapolation. Eur Radiol 2011;21(7): 1424-1429

7 Pessis E, Campagna R, Sverzut JM, et al. Virtual monochromatic spectral imaging with fast kilovoltage switching: reduction of metal artifacts at CT. Radiographics 2013;33(2):573-583

8 Meinel FG, Bischoff B, Zhang Q Bamberg F, Reiser MF, Johnson TR. Metal artifact reduction by dual-energy computed tomography using energetic extrapolation: a systematically optimized protocol. Invest Radiol 2012;47(7):406-414

9 Potter HG, Foo LF. Magnetic resonance imaging of joint arthroplasty. Orthop Clin North Am 2006;37(3):361-373, vi-vii

10 U.S. Food and Drug Administration. Information about soft tissue imaging and metal ion testing. Available at: http://www. fda.gov/MedicalDevices/ProductsandMedicalProcedures/Impl antsandProsthetics/MetalonMetalHipImplants/ucm331971. htm. Accessed March 11, 2013

11 Hayter CL, Koff MF, Shah P, Koch KM, Miller TT, Potter HG. MRI after arthroplasty: comparison of MAVRIC and conventional fast spinecho techniques. AJR Am J Roentgenol 2011;197(3):W405-W411

12 Koch KM, Lorbiecki JE, Hinks RS, King KF. A multispectral threedimensional acquisition technique for imaging near metal implants. Magn Reson Med 2009;61(2):381-390

13 D'Lima DD, Urquhart AG, Buehler KO, Walker RH, Colwell CW Jr. The effect of the orientation of the acetabular and femoral components on the range of motion of the hip at different headneck ratios. J Bone Joint Surg Am 2000;82(3):315-321 
14 Barrack RL, Lavernia C, Ries M, Thornberry R, Tozakoglou E. Virtual reality computer animation of the effect of component position and design on stability after total hip arthroplasty. Orthop Clin North Am 2001;32(4):569-577, vii

15 Barrack RL. Dislocation after total hip arthroplasty: implant design and orientation. J Am Acad Orthop Surg 2003;11(2):89-99

16 Miller TT. Imaging of hip arthroplasty. Semin Musculoskelet Radiol 2006;10(1):30-46

17 De Haan R, Pattyn C, Gill HS, Murray DW, Campbell PA, De Smet K. Correlation between inclination of the acetabular component and metal ion levels in metal-on-metal hip resurfacing replacement. J Bone Joint Surg Br 2008;90(10):1291-1297

18 De Haan R, Campbell PA, Su EP, De Smet KA. Revision of metal-onmetal resurfacing arthroplasty of the hip: the influence of malpositioning of the components. J Bone Joint Surg $\mathrm{Br} 2008 ; 90$ (9):1158-1163

19 Morscher EW. Cementless total hip arthroplasty. Clin Orthop Relat Res 1983;(181):76-91

20 Moore MS, McAuley JP, Young AM, Engh CA Sr. Radiographic signs of osseointegration in porous-coated acetabular components. Clin Orthop Relat Res 2006;444:176-183

21 Lindahl H, Garellick G, Regnér H, Herberts P, Malchau H. Three hundred and twenty-one periprosthetic femoral fractures. J Bone Joint Surg Am 2006;88(6):1215-1222

22 Goldring SR, Schiller AL, Roelke M, Rourke CM, O'Neil DA, Harris $\mathrm{WH}$. The synovial-like membrane at the bone-cement interface in loose total hip replacements and its proposed role in bone lysis. J Bone Joint Surg Am 1983;65(5):575-584

23 Bosetti M, Massè A, Navone R, Cannas M. Biochemical and histological evaluation of human synovial-like membrane around failed total hip replacement prostheses during in vitro mechanical loading. J Mater Sci Mater Med 2001;12(8):693-698

24 Berger R, Fletcher F, Donaldson T, Wasielewski R, Peterson M, Rubash H. Dynamic test to diagnose loose uncemented femoral total hip components. Clin Orthop Relat Res 1996; (330)115-123

25 Gruen TA, McNeice GM, Amstutz HC. "Modes of failure" of cemented stem-type femoral components: a radiographic analysis of loosening. Clin Orthop Relat Res 1979; (141) 17-27

26 DeLee JG, Charnley J. Radiological demarcation of cemented sockets in total hip replacement. Clin Orthop Relat Res 1976; (121):20-32

27 Engh CA Jr, McAuley JP, Sychterz CJ, Sacco ME, Engh CA Sr. The accuracy and reproducibility of radiographic assessment of stressshielding. A postmortem analysis. J Bone Joint Surg Am 2000;82-A (10):1414-1420

28 Manaster BJ. From the RSNA refresher courses. Total hip arthroplasty: radiographic evaluation. Radiographics 1996;16(3): 645-660

29 Engh CA, Griffin WL, Marx CL. Cementless acetabular components. J Bone Joint Surg Br 1990;72(1):53-59

30 Engh CA, Massin P, Suthers KE. Roentgenographic assessment of the biologic fixation of porous-surfaced femoral components. Clin Orthop Relat Res 1990;(257):107-128

31 Harris WH, McCarthy JC Jr, O'Neill DA. Femoral component loosening using contemporary techniques of femoral cement fixation. J Bone Joint Surg Am 1982;64(7):1063-1067

32 Malchau H, Kärrholm J, Wang YX, Herberts P. Accuracy of migration analysis in hip arthroplasty. Digitized and conventional radiography, compared to radiostereometry in 51 patients. Acta Orthop Scand 1995;66(5):418-424

33 Bauer TW, Schils J. The pathology of total joint arthroplasty. II. Mechanisms of implant failure. Skeletal Radiol 1999;28(9): 483-497

34 Dumbleton JH, Manley MT, Edidin AA. A literature review of the association between wear rate and osteolysis in total hip arthroplasty. J Arthroplasty 2002;17(5):649-661
35 McKellop HA. Bearing surfaces in total hip replacements: state of the art and future developments. Instr Course Lect 2001;50: 165-179

36 Baxter RM, MacDonald DW, Kurtz SM, Steinbeck MJ. Characteristics of highly cross-linked polyethylene wear debris in vivo. J Biomed Mater Res B Appl Biomater 2013;101(3):467-475

37 Maloney WJ, Galante JO, Anderson M, et al. Fixation, polyethylene wear, and pelvic osteolysis in primary total hip replacement. Clin Orthop Relat Res 1999;(369):157-164

38 Claus AM, Engh CA Jr, Sychterz CJ, Xenos JS, Orishimo KF, Engh CA Sr. Radiographic definition of pelvic osteolysis following total hip arthroplasty. J Bone Joint Surg Am 2003;85-A(8):1519-1526

39 Walde TA, Weiland DE, Leung SB, et al. Comparison of CT, MRI, and radiographs in assessing pelvic osteolysis: a cadaveric study. Clin Orthop Relat Res 2005;(437):138-144

40 Schmalzried TP. Metal-metal bearing surfaces in hip arthroplasty. Orthopedics 2009;32(9):

41 Amstutz HC, Le Duff MJ, Campbell PA, Wisk LE, Takamura KM. Complications after metal-on-metal hip resurfacing arthroplasty. Orthop Clin North Am 2011;42(2):207-230, viii

42 Huber M, Reinisch G, Trettenhahn G, Zweymüller K, Lintner F Presence of corrosion products and hypersensitivity-associated reactions in periprosthetic tissue after aseptic loosening of total hip replacements with metal bearing surfaces. Acta Biomater 2009;5(1):172-180

43 Campbell P, Ebramzadeh E, Nelson S, Takamura K, De Smet K, Amstutz HC. Histological features of pseudotumor-like tissues from metal-on-metal hips. Clin Orthop Relat Res 2010;468 (9):2321-2327

44 Nawabi DH, Gold S, Lyman S, Fields K, Padgett DE, Potter HG. MRI predicts ALVAL and tissue damage in metal-on-metal hip arthroplasty. Clin Orthop Relat Res 2013; January 26 ( Epub ahead of print)

45 Hart AJ, Sabah SA, Bandi AS, et al. Sensitivity and specificity of blood cobalt and chromium metal ions for predicting failure of metal-on-metal hip replacement. J Bone Joint Surg Br 2011;93 (10):1308-1313

46 Bauer TW, Parvizi J, Kobayashi N, Krebs V. Diagnosis of periprosthetic infection. J Bone Joint Surg Am 2006;88(4):869-882

47 Palestro CJ, Love C, Tronco GG, Tomas MB, Rini JN. Combined labeled leukocyte and technetium 99m sulfur colloid bone marrow imaging for diagnosing musculoskeletal infection. Radiographics 2006;26(3):859-870

48 Karchevsky M, Schweitzer ME, Morrison WB, Parellada JA. MRI findings of septic arthritis and associated osteomyelitis in adults. AJR Am J Roentgenol 2004;182(1):119-122

49 Plodkowski AJ, Hayter CL, Miller TT, Nguyen JT, Potter HG. Lamellated hyperintense synovitis: potential MR imaging sign of an infected knee arthroplasty. Radiology 2013;266(1):256-260

50 Pike J, Davidson D, Garbuz D, Duncan CP, O’Brien PJ, Masri BA. Principles of treatment for periprosthetic femoral shaft fractures around well-fixed total hip arthroplasty. J Am Acad Orthop Surg 2009;17(11):677-688

51 Duncan CP, Masri BA. Fractures of the femur after hip replacement Instr Course Lect 1995;44:293-304

52 Pritchett JW. Fracture of the greater trochanter after hip replacement. Clin Orthop Relat Res 2001;(390):221-226

53 Cook SM, Pellicci PM, Potter HG. Use of magnetic resonance imaging in the diagnosis of an occult fracture of the femoral component after total hip arthroplasty. A case report. J Bone Joint Surg Am 2004;86-A(1):149-153

54 Thomas BJ. Heterotopic bone formation after total hip arthroplasty. Orthop Clin North Am 1992;23(2):347-358

55 Brooker AF, Bowerman JW, Robinson RA, Riley LH Jr. Ectopic ossification following total hip replacement. Incidence and a method of classification. J Bone Joint Surg Am 1973;55(8):1629-1632 
56 Magid D. Preoperative interactive 2D-3D computed tomography assessment of heterotopic bone. Semin Arthroplasty 1992;3(3): 191-199

57 Lachiewicz PF. Abductor tendon tears of the hip: evaluation and management. J Am Acad Orthop Surg 2011;19(7):385-391

58 Douis H, Dunlop DJ, Pearson AM, O'Hara JN, James SL. The role of ultrasound in the assessment of post-operative complications following hip arthroplasty. Skeletal Radiol 2012;41(9): 1035-1046
59 Connell DA, Bass C, Sykes CA, Young D, Edwards E. Sonographic evaluation of gluteus medius and minimus tendinopathy. Eur Radiol 2003;13(6):1339-1347

60 Pfirrmann CW, Notzli HP, Dora C, Hodler J, Zanetti M. Abductor tendons and muscles assessed at MR imaging after total hip arthroplasty in asymptomatic and symptomatic patients. Radiology 2005;235(3):969-976

61 Lonner JH, Lotke PA. Aseptic complications after total knee arthroplasty. J Am Acad Orthop Surg 1999;7(5):311-324 